\title{
Flexible automatic detection for security management of dispatching data network
}

\author{
Xianrui Yang ${ }^{1}$, Yuming Liu' ${ }^{1}$, Jiehong Wang ${ }^{1}$, Zhao Yao ${ }^{1}$, Yanping Zhou ${ }^{1}$ and Shucun Fu ${ }^{*}$
}

\begin{abstract}
Power grid dispatching is among the forefront issues in the power industry for it can highly influence the efficiency of electricity-related industries. At present, power grid dispatching is usually managed manually, which is quite time-consuming with the continuous growth of scale and complexity of electric power systems. To mitigate the time cost of power grid dispatching, an automatic power grid dispatching system is proposed. The system contains devices configuration detection, flexible and automatic device detection and vertical encryption to ensure the efficiency, adaptability and security of power gird dispatching. In this paper, modeling and algorithm details of the automatic power grid dispatching system are presented, and the effectiveness and efficiency of the proposed system was evaluated with power grid data in Kunming, China.
\end{abstract}

Keywords: Power grid dispatching, Automatic detection, Data network

\section{Introduction}

With the rapid progress of technology and social economy, electricity has become a necessary foundation for the development of the society. In social production, residents' life and basic services, electricity has become an essential and fundamental energy. As the dependence of society on electricity is increasing, the capacity of electric power system is correspondingly becoming more demanding [1].

Generally, the electric power system undertakes vital responsibility of power production, transmission and distribution in the whole society. In order to realize the safe and stable power supply and the high quality of power service, it is necessary to avoid the occurrence of major safety accidents [2]. The important position and role of electric power system put forward high requirements and standards for the safety of power production. If the power system has safety risks in the production and transmission periods, it will cause serious damage to the proper operation of the whole society, including safety accidents,

*Correspondence: shucunfu@163.com

${ }^{2}$ Nanjing University of Information Science and Technology, China No.219

Ningliu Road, 210044 Nanjing, China

Full list of author information is available at the end of the article and serious economic losses [3]. During the operation of the electric power system, the first thing to meet is to stably provide the end users with safe and high-quality power supply, to promote the rapid development of social economy, and to realize the economic benefit demand of the power industry $[4,5]$. In the current environment of electric power system development, the scale and complexity of the power grid are getting higher, and the types and quantity of basic hardware infrastructures included in it have increased dramatically. In addition, the security conditions of the power grid are also becoming more demanding. Thus, the automatic power grid dispatching system is urgently needed to accomplish the tasks effectively [6, 7].

The automatic power grid dispatching system is based on the information acquisition subsystem and command execution subsystem. It is a set of complex software and hardware systems containing multiple computer software and hardware equipment, which is used to provide status monitoring, data collection, communication support and other functions for the power company's power grid management $[8,9]$.The automatic power grid dispatching system is a management system that assists in 
decision-making of power grid operation safety management, daily maintenance management, and electric power system marketing business management. According to its distribution characteristics and realized functions, it can be divided into certain levels, and its higher level functions are often built on certain basic functions [10, 11]. The devices and system of automatic power grid dispatching play important roles in the development of electric power system. With the continuous advancement of science and technology, the automation level of automatic power grid dispatching system has also been greatly improved [12]. It has developed from a single relay automatic protection device to a comprehensive control platform based on network communication technology, automation technology and computer software technology [13]. The control platform can realize the remote monitoring and controlling of power grid infrastructures through the power dispatching master station, realizing the important evolution from the manual management to the automatic management of the power grid, and greatly improve the comprehensive operation ability of the power management department on this basis [14].

At present, the automatic power grid dispatching system is based on the underlying monitoring and control hardware facilities, and realizes the automatic remote monitoring of the power grid operation state by comprehensively using the network communication technology, computer software technology, information technology and database technology [15]. At the same time, it realizes the remote control of underlying security protection hardware through the central control software system or the main station system by unattended methods. The remote control can realize the safe and stable operation of the power grid, ensure the production and transmission of power, and provide basic data and technical support to the power management personnel [16]. The support that provided including the security and economic analysis, load condition prediction, power grid fault location and analysis and remote dispatching command $[17,18]$. In this way, the working pressure of power grid dispatching can be reduced, and the overall operation efficiency of the power grid can be improved. Automatic power grid dispatching system is an important part of electric power system in modern environment, and the quality of the system is directly related to the level of automation $[19,20]$.

The installation and deployment of automatic power grid dispatching system, which is usually enabled by the dispatching data network, can provide decision-making reference and basis for power production and other management work for the decision-making layer of the power management department $[21,22]$. The dispatching data network enables the transmission of automation information, dispatching command instructions, relay protection and safety automation device control information in the power grid dispatching system [23]. It is an important technical means to improve the overall operation quality and efficiency of electric power system. The emergence of the automatic power grid dispatching system has transformed the traditional manual management mode of power grid dispatching, into the information technologybased automatic management mode, which has improved the overall technical content and accuracy of power grid dispatching work $[24,25]$. The installation and deployment of power grid dispatching automation software platform is a novel upgrade to the traditional mode of power grid dispatching work, which is in line with the current construction and management requirements of the new power grid in China [26]. With the platform that deployed, the development goal of safe and stable operation of the basic electric power system can be achieved, and the economic benefits of grid operation can be improved. Specifically, the key contributions of this paper are as follows:

1) Design an automatic detection scheme for devices configuration strategy, which can quickly obtain the internal communication strategy of the equipment and replace the manual analysis of problems. Then, the latest status and problems of all equipment are displayed in report forms in a short time.

2) A flexible automatic detection technology is proposed to adapt to different devices security protection configurations and strategies of the power dispatching data network. This technology is extensible, programmable and devices adaptable.

3) The dispatching data network is divided into several virtual areas, which are allocated according to the load balancing strategy. Then, distributed technology is used to improve the security of the dispatch data network.

The remainder of this paper is organized as follows: In "Related work" section, we review the peer research and work. "System model" section describes the details of power grid dispatching automation system. The system implementation, experiments and their results and analysis are covered in "System implementation and experimental analysis" section. Finally, we conclude our work in "Conclusion" section.

\section{Related work}

With the development of technology, the production and transmission process are controlled by the management system instead of the staffs. The reliability and efficiency of the management system are of great significance to the company. Research on control systems has attracted many experts and scholars in this field and there are still many problems to solve [27].

Management system is widely used in many aspects. Okami et al. [28], set up a model to analyze the transforming health-information system. In [29], Qin et al. designed 
a production information and managing system which depends on Internet of Things. This network architecture performs well and has great reliability and safety. Levendovszky et al. [30], mix runtime solution with practical design to manage constrained resources.

Many works have done to obtain the suitable electric systems in different situations. In [31], Dmitriev et al. proposed a object-oriented model to identify the defects appear as well as the failures. They also determined the methods which can offer reliable power supply in this model. What's more, Hill et al. introduced an Automated Stability Assessment Tool which can provide a easy way to analyze the stability of an electrical power system in [32]. This method can evaluate the impact of each system parameter on the stability of the system. Kryukov et al. [33], put forward a new method which monitors the redundant. Many optimization strategies are proposed in this paper to deal with different situations [34].

Security management plays a significant role in the dispatching data network. Without proper security management, the power grid dispatching system can be easily hacked, which will cause not only economic loss but even casualties. Many researchers studied security management in different scenarios. Zhou et al. in [35] researched on the task scheduling onto a heterogeneous multiprocessor system while considering the quality of security. In [36], a privacy enhancement scheme was proposed by Zhang et al. to reduce the risk of exposure of user' information to untrusted location service provider. To handle the problem of user information leakage when distributed service recommendations are made, a recommendation approach named DistSR Amplify-LSH was proposed in [37]. And in [38], Qi et al. concerned the security of user privacy into recommendation domain to accommodate users with location-aware and latency-sensitive recommendation services.

Since a number of indicators are set up in electrical power system to evaluate its performance, multi-objective optimization algorithms are applied in such situations to schedule the system. In [39, 40], Cao et al. used NSGAIII to determine scheduling strategy of workflow. Xu et al. applied NSGA-II in cloudlet-based mobile cloud to control the computation offloading in [41]. The multiobjective optimization algorithm performed well in terms of improving all the indicators of the whole system instead of only raising one of the indicators. In the electrical power system, efficiency and reliability both are supposed to be taken into consideration and neither of them can be ignored.

Among the previous works, there were some researchers, such as Okami and Qin, who focused on management system in terms of health-information system and production information system. However, few researchers combined security management and dispatching data network in power grid dispatching system. How to ensure the efficiency, adaptability and security of power gird dispatching still remains a challenge. Because of the significance of electricity in the development of the society and the importance of avoiding occurrence of major safety accidents in power production, it is urgent and necessary to build a dispatching data network with flexible automatic detection for security management.

\section{System model}

This section first describes the business of grid dispatching system. Then, the system functional requirements are analyzed and given. Next, the system conceptual design model is discussed. Finally, we present the design of the grid dispatching system in detail.

\section{System business description}

Grid dispatching automation, which is usually developed in computer software, mainly refers to providing a unified management platform for grid dispatching staff in the form of automation based on a series of grid management tools, equipment, and software of power supply companies. The power grid dispatching automation system is mainly used to realize the acquisition and analysis of power grid operating status data remotely in the power supply company's internal private network environment, and to realize the inversion operation of abnormal power grid accidents by means of virtualization and automation.

Therefore, for the power grid dispatching automation system, its research and development work mainly need to realize the concentration and integration of the current basic grid information. Based on this, the existing power grid operation data management platform of the power supply company is used to obtain the power grid operating status information, such as SCADA system.

With the obtained grid operation status data recorded, analyzed and compared to the safe grid operation threshold set by the grid dispatchers, abnormal situations are alerted to grid dispatchers. In the meantime, based on the recorded grid operating state data, the fault inversion processing of the abnormal state of the grid is realized. The system mainly obtains real-time operating status data of hardware such as substations, transmission lines, and power equipment in the grid by data communication with SCADA and other grid state management software platforms.

During the system's internal recording of the data, abnormal analysis and detection of data are done. When abnormal conditions are detected, the power grid dispatcher is alerted. Based on the abnormal data information and the operating status information of the power grid, the power grid dispatchers are provided with auxiliary reference functions of power grid dispatching 
management within the system. Therefore, automation of the processing of power grid dispatching operations is built to some extent.

\section{System functional requirements}

In software development, requirement analysis is one of the most critical and basic tasks. Its main tasks include system business environment analysis, functional requirements analysis, data flow analysis, and other performance and security analysis. Requirement analysis is an important reference for the design and implementation of the software system. In the grid dispatching system, for the two different types of system users: grid dispatch managers and system administrators, the system proposes five areas of demand: data collection functions, grid dispatch work management functions, data report management functions, dispatch parameter setting management functions, and system management functions. Among them, the first four requirements are for grid dispatch managers, and the last is for system administrators. The use case structure of the grid dispatch automation system is shown in Fig. 1.

As shown in Fig. 1, the grid dispatchers utilize the data acquisition, power grid dispatch management, datagram management, and scheduling data parameters setting management functions to automate power management scheduling. The system administrator utilizes the system management function to realize the operation and maintenance management of the system. This section analyzes and explains the use cases of each functional requirement of the system in detail.

The parameter setting management function in the system refers to the setting and management of systemrelated parameters in the system's back-end database during the operation of the system. Parameter setting functions mainly include system operation settings, report management settings, remote signal telemetry power settings, data type settings, and calculation volume settings. System operation settings enable staff members to edit the relevant data set information tables in the database and provide real-time display in the foreground of the screen according to the specific settings of the managers. The report management settings enable managers to edit specific types of data reports in the system, including adding report types and other custom operations. The remote signal telemetry power settings allow managers to modify and set the remote signal telemetry and other power parameters of all current stations in the system online. It is the significant basic information management function of the system. The data type settings need to automatically set all relevant data types during the initialization process, including related data types such as telemetry, power and other relevant data types. In the specific data type settings, you can follow the system's hardware ID

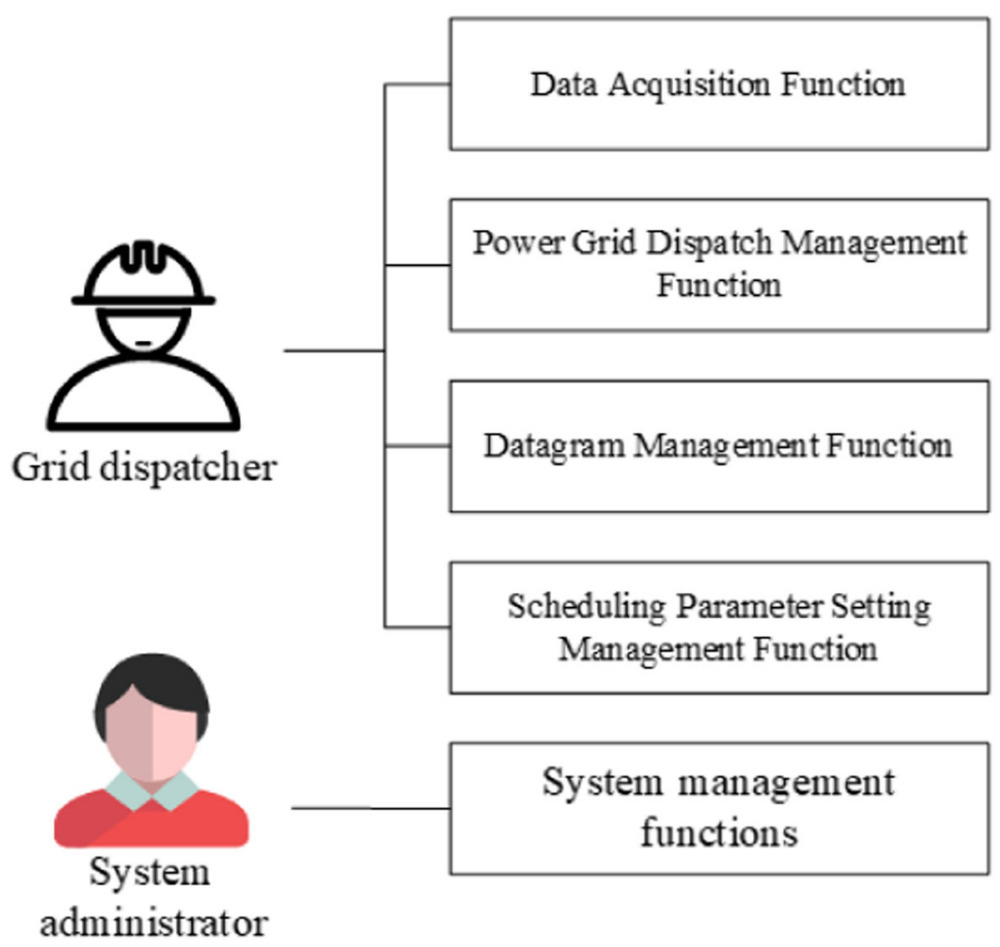

Fig. 1 Use case structure diagram of power grid dispatch automation system 
number and the corresponding Chinese and English Names are combined, and managers can add or edit data types in the system. The calculation capacity setting function of the system means that the manager can dynamically set the expression mode of the relevant calculation quantity in the system.

Basic data collection function refers to the collection and subsequent processing station site operating data information, as in the current SCADA system to collect data grid operation can not achieve real-time requirements, there is no law to meet the aging power grid dispatching work Sexual requirements. Therefore, basic data collection function is an important function item dispatching automation system functional requirements, the system needs to be able from the front end acquires operating data of the scene RTU apparatus, by serial communication and a network communication technology based data operation the obtained information to Processing in the master station system. In the data processing process, the received data information needs to be encoded and parsed first, and a corresponding data information analysis model is constructed for analysis and processing. Finally, the analysis result and the original monitoring data are stored in the background database system.

By analyzing the business process of power grid dispatching, it can be seen that the power grid dispatching functions the system needs to implement mainly include SOE event management functions, alarm information management functions, telemetry and remote signal parameter management functions, remote management functions and control commands. Among them, SOE event management is one of the core functions of the system. By querying, retrieving and managing of the SOE in the function, managers can have access to the running status and the historical status of the site.

The report management function of the system mainly means that the system can perform related report management operations based on the business data stored in the back-end database. It can obtain the corresponding report file and display it in the interface according to the actual needs of the management staff. The managers can customize the operation of the specific format of the table, additionally. In the meanwhile, the system also needs to provide online printing capabilities for management reporting.

The system management function refers to the related functional requirements that must be met in order to ensure the stable operation of the system and the rationality and security of business processing of the entire system. It mainly includes user information management, site information and device information management, system background database backup.

\section{System conceptual design model}

The power grid dispatching automation system utilized vertical encryption to enhance the safety of the system. With vertical encryption method and the demand analysis work of the power grid dispatching automation system, the overall topology design of the power grid dispatching automation system is shown in Fig. 2.

As shown in Fig. 2, the power grid dispatching automation system with vertical encryption has four layers: controller, service, dao and basics. The controller layer provides users with Equipment security baseline management functions and network active detection functions to detect and reject insecure login requests and virus. The service realizes six methods, i.e., user identity authentication method, user management method, rule management method, command management method, data analysis method and data collection method, for the control layer to provide users with a variety of services. The dao layer is composed of two parts, i.e., User database and vertical encryption database. Vertical encryption database is further made up by device rule command and data acquisition and analysis database. This layer stores the significant information about users and vertical encryption. The bottom layer is the basic layer, which offers some basic function for the upper layers, e.g., user authentication, IP range detection, test site analysis and template check.

\section{Detailed system design System function design}

The overall functional configuration of dispatching automation system is based on the B/S network structure development patterns. MVC pattern design concept is used in the logical structure design. The system is divided into three layers, i.e., functional hierarchy interactive layer, logical layer, and hierarchical data. Due to the current development of distributed application systems, the MVC hierarchical design pattern is a more commonly used design pattern. It can divide and package system functions from the overall logical architecture level and is more convenient to adopt object-oriented and modular design and development. The specific contents included in the functional hierarchy of this system are as follows:

(a) The interaction layer contains client software that provides the user-machine function operation interaction interface.

(b) The logic layer is composed of business data collection and processing, power grid dispatch management, data report management, parameter setting management, and system management in the master station system. It is responsible for the realization of the core business of the system. 


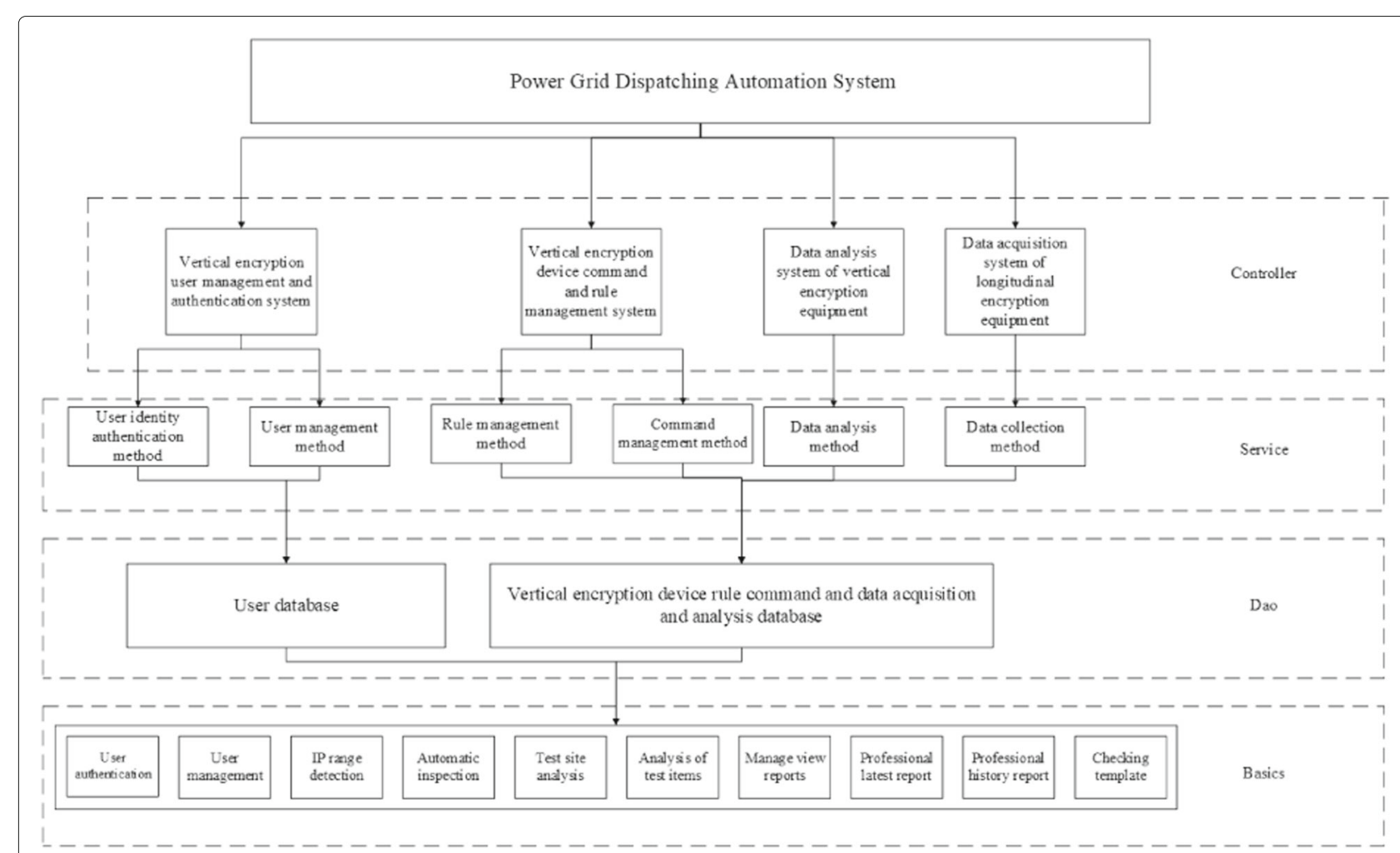

Fig. 2 The overall topology design of the power grid dispatching automation system

(c) There is a systematic background database system at the data layer of the system, which is mainly responsible for the storage and overall management of various business data and other information of the system.

At the same time, by summarizing and researching the software architecture of the system and the analysis of the functional requirements of the system, it can be obtained that the system mainly includes 5 modules, i.e., data acquisition function module, grid dispatching function module, report management function module, parameter setting function module, and system management module. Software architecture of the system is shown in Fig. 3. The function page is mainly used to provide system management with interactive dispatching page interfaces and is physically deployed with C\# logic background components together in the IIS Web service management component. However, from a logical level, it is a functional component in the interaction interface.

According to the functional structure design shown in Fig. 3, the functional structure of the dispatching automation system includes data acquisition module, power scheduling module, report management module, system parameter setting module and system management module. In the functional structure design of the system, the system parameter setting module proposed in the demand analysis is merged into the grid dispatch management function.

The data acquisition module includes communication parameter setting, data analysis, interface display, data communication time correction and other functions. Power scheduling module is the core function of the module. Based on power data acquisition, event information processing in power dispatching, alarm management, telemetry/remote communication parameter management, remote control/adjustment management and device control commands management are realized.

Report management module includes the functions of management of the data reports generated during the process of grid dispatching, such as the default management report creation, custom report creation and online print management of the above-mentioned report forms.

System management module includes user information and department information management, grid site and equipment information management, system database management system safety management and other functions.

\section{System database design}

The System back-end database system uses Microsoft SQL Server 2000 relational database management system. 


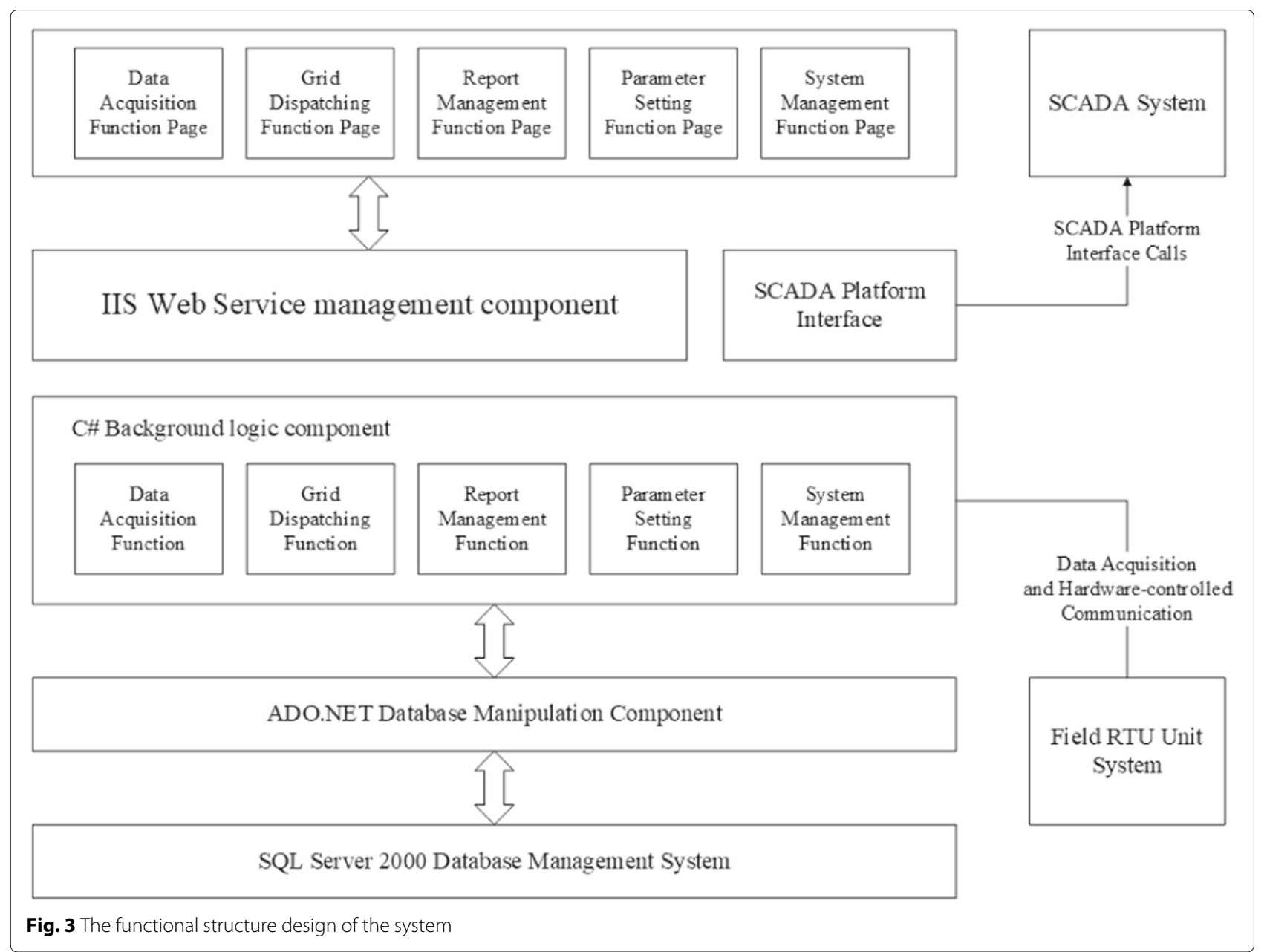

With the analysis of the system business environment, it can be inferred that the system back-end database entities include a telemetry point, communication parameters, remote data, report information, computing information, telecontrol RTU equipment information, SOE event information and other data entities.

The database is composed of five data tables: calculation table, report information table, data operation event table, telemetry data table, and remote communication system table. Calculation table records the number of calculations in the system back-end database. Report information table stores the information on data report forms, e.g., the name of the data report form, the storage path. The information about historical operations in the database is recorded in the operation event table. Telemetry data table mainly records the telerecordings. The Remote communication system table stores remote communication records in the system. The number of the types of data table can increase when new data table is needed. The details of the data tables is designed according to the demands of the production process. In general, the data tables are not supposed to be too large to take up too much space.

\section{System implementation and experimental analysis System implementation Interface implementation}

The master station system also includes a functional operation interface for interacting with the existing SCADA system. Use the existing information management resource platform of the power sector to reduce system development and maintenance costs. Master station The system also uses the internal power private network to connect with the information management platform of the municipal bureau's power management department to provide power management. The management department provides decision-making reference and guidance for grid management. The functional interaction interface model design between the system and other platforms and 


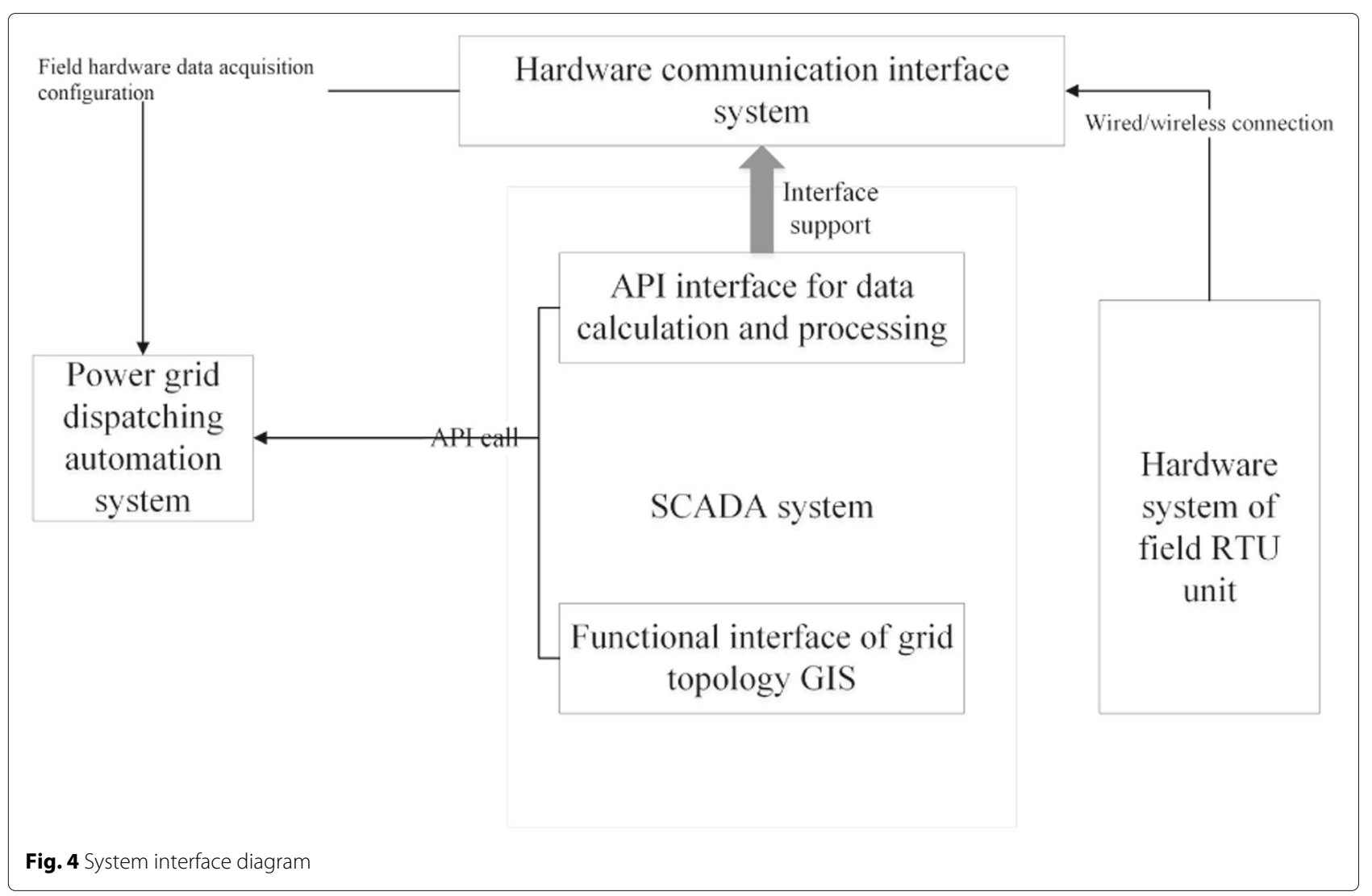

hardware devices such as the SCADA system platform is shown in Fig. 4.

As shown in the interface Fig. 4, the entire system consists of the on-site RTU unit hardware system, the hardware communication interface system, the data calculation processing API interface, the grid topology GIS function interface, and the grid dispatch automation system. First, the on-site RTU unit hardware accesses the hardware communication interface through a wired or wireless connection, and provides on-site hardware operating data, status, and environmental information. Next, the hardware communication system is connected to the SCADA system for numerical calculations through a data calculation processing interface. At the same time, the data calculation function provides an interface for the grid dispatch automation system to perform data calculations. The grid topology GIS function in the SCADA system will also provide an interface to the grid dispatch automation system.

First of all, the on-site RTU unit hardware accesses the hardware communication interface through a wired or wireless connection, and provides on-site hardware operating data, status, and environmental information. The SCADA system needs the data from the hardware communication system for numerical calculations. Therefore, the hardware communication system is connected to the
SCADA system through a data calculation processing interface. At the same time, the data calculation function provides an interface for the grid dispatch automation system to perform data calculations. The grid topology GIS function in the SCADA system will also provide an interface to the grid dispatch automation system. These parts work together to make the system go well when facing all kinds of situations. If some accidents happen when the system works, the part which does not receive the correct data would send the warnings and the staff can get involved and solve the problems according to the historical record.

In this way, the communication between the various systems through the interface provides the necessary guarantee for the operation of the grid dispatch automation system. The on-site RTU unit hardware system provides the detected grid operation data (such as network configuration, line configuration, and encryption status), and is uniformly received and organized by the hardware communication interface.

(1) The RTU hardware communication interface of this system borrows the communication system of the SCADA system, and performs hardware communication parameter setting and communication process control in the power grid dispatching automation system. There is no need to redeploy and install intermediate hardware 
communication link resources. The RTU unit can access the system through a wired network or a wireless network. The system interacts with the RTU unit through the RTU unit hardware communication interface already deployed in the SCADA system.

(2) The analysis and calculation interface of the on-site data collection results is based on the function development kits such as grid cycle calculation, voltage and power calculation, load flow analysis calculation, load calculation, abnormality and fault detection calculation provided in the SCADA system, and the system is implemented through API calls to realize the calculation processing function of the parsed data result.

(3) The graphical processing interface of the power grid topology refers to the display function of the power grid topology in this system, which needs to display the topology of the power grid in this system through the GIS geospatial function component in the SCADA system. In the specific function processing, the GIS subsystem in the SCADA system can be directly integrated into the system.

\section{Physical design of database}

Database logic design refers to the analysis of relevant processing processes in the software system business environment, from which the data entities contained in the back-end database of the system and the relationships between the data entities are obtained. Normally. E-R diagrams (Entity-Relationship Diagram, entity-relationship diagram) in unified modeling technology. Logic design work of row database. The background database system of this system uses the Microsoft SQL Server 2000 relational database management system. Through analysis of the system's business environment, it can be obtained that the data entities in the system's back-end database mainly include remote measurement points, communication parameters, remote signaling data, report information, calculation information, remote RTU equipment information, and SOE data entities such as event information.

The E-R diagram is used to represent the above data entities and the relationships between them, and the database E-R diagram shown in Fig. 5 can be obtained. The E-R diagram shows some entities in the system's back-end database and their relationship information. The scale of the system's back-end database is relatively large, limited to the length of the paper, and detailed information about some entities, attributes and relationships.

\section{Experiment and analysis}

The "Management View Report" is mainly divided into "Project Analysis Overview", "Alarm Classification Overview" and "Historical Alarm Overview".

\section{Project analysis overview}

The detection status of system configuration, network configuration, route configuration, tunnel configuration, policy configuration, encrypted state, tunnel state and strategy status of all devices are shown in Fig. 6. From the figure, it is intuitive that most of the detection information of power grid equipment is in normal state, especially the route configuration, tunnel configuration and tunnel state. Basically, all the equipment was in normal state, indicating that the three configurations are in good condition. In addition, for the network configuration and strategy status, some devices are in the common state, indicating that the problem is not serious and does not hinder the normal operation of the device. However, for system configuration, policy configuration and strategy status, there are many devices in a series state, which seriously affects the normal operation of the equipment, indicating the need for timely maintenance and repair. This result shows the proposed power grid dispatching system's ability to analyze the current running state of the project. With the proposed system, it is expediently to obtain and the check the status of different configurations, so the dispatchers can adjust and repair the according system configuration based on current status easily.

At the same time, the project analysis overview report also supports the function of hiding a detection analysis result classification. As shown in Fig. 7, Click the "normal" icon in the upper right corner to gray it, and the report will automatically hide the check. In general, we will choose to hide the devices in normal state and keep the data of devices in serious and common state. For the function of our system is to detect the data and repair the damaged devices in time, hiding the devices in normal state is able to enhance the readability of the analysis report and facilitate the maintenance of the devices. In other cases, for example, if we need to view the number of available devices, we could also choose to hide the data of the series status devices to intuitively get the number of available devices from the report.

\section{Alarm classification overview}

The "Alarm Classification Overview" report uses the form of a pie chart to analyze the detection results in three types: "Serious", "Common", and "Normal". The alarm classification is displayed in Fig. 8. From the alarm classification overview, it can be seen that most of the equipment that can operate normally accounts for $71.88 \%$, indicating that the power grid system is in good condition. However, the proportion of devices in common state is small, only $9.07 \%$ of them are in common state, which will not have a great impact on the normal operation of the device. Finally, $19.05 \%$ of the equipment is in a series state, which indicates that the operation of the equipment is seriously disturbed or hindered, so it is necessary to carry out 


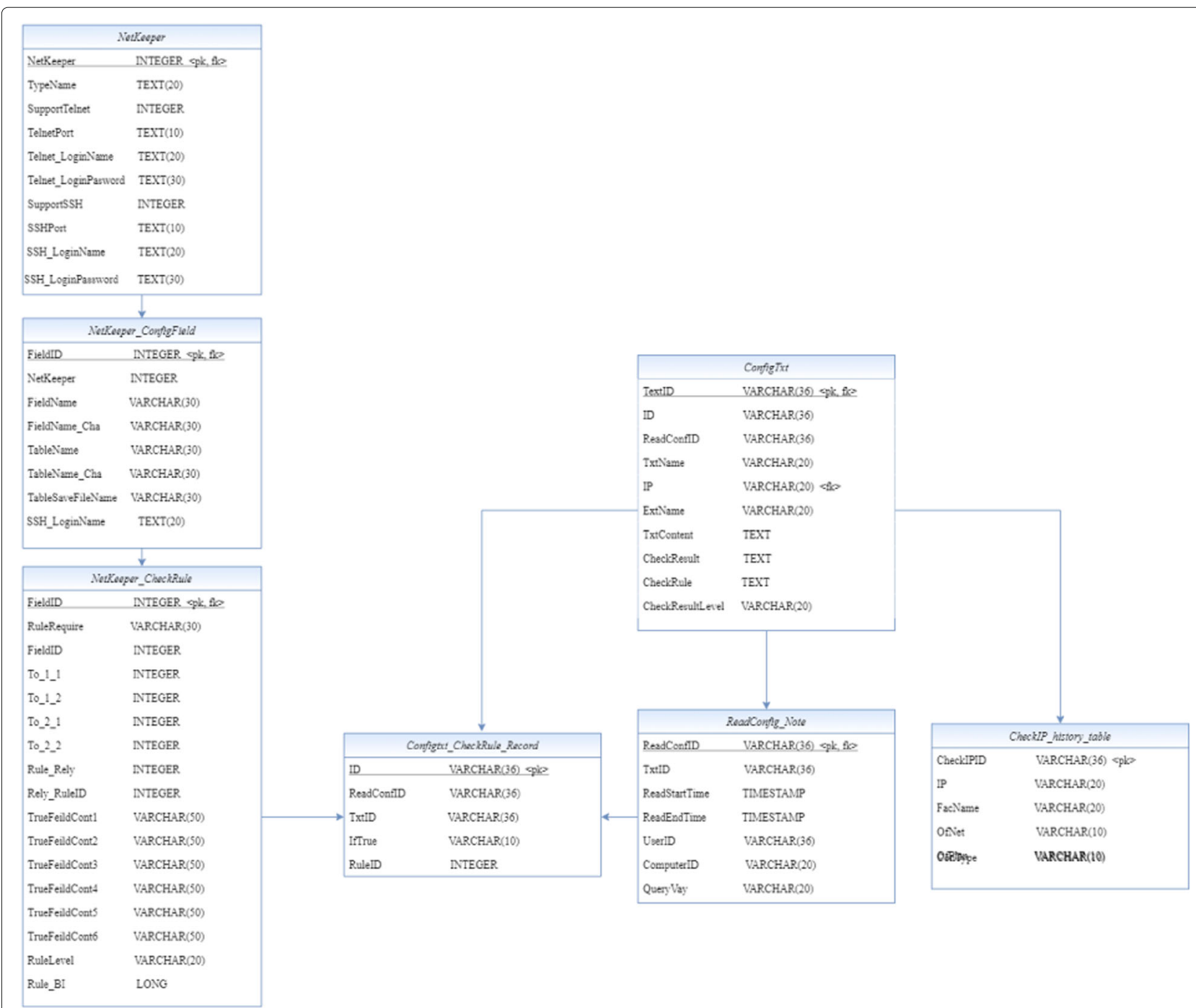

Fig. 5 Physical design of database

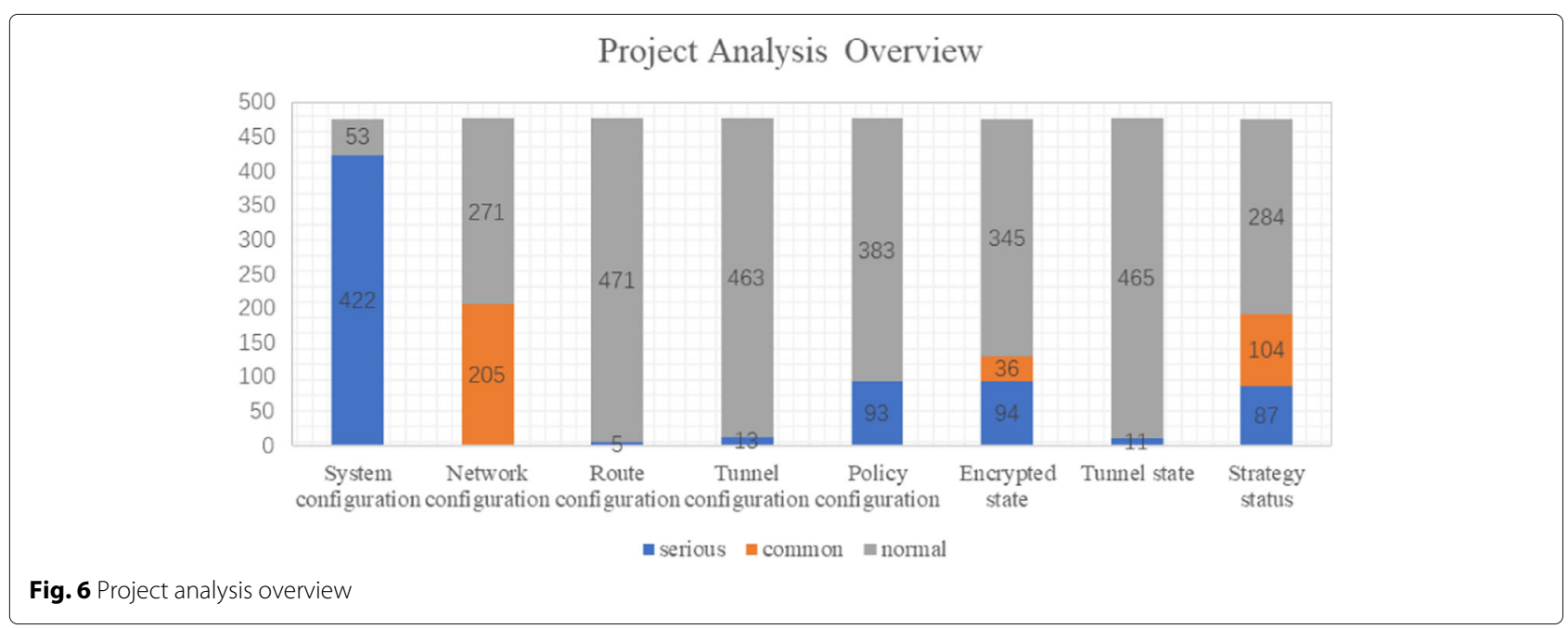




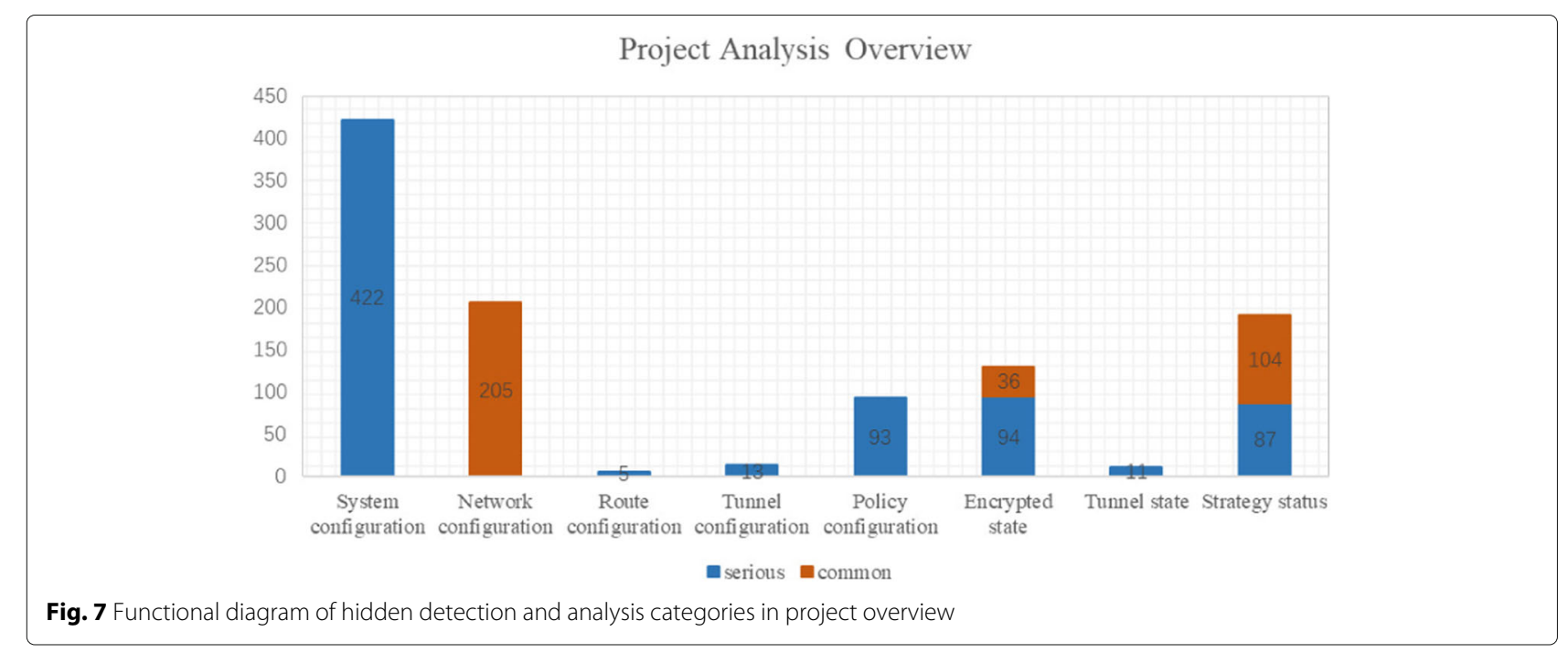

maintenance and repair in time to ensure the normal operation of the whole power grid system.

\section{Historical alarms overview}

As shown in the Fig. 9, the historical alarms overview shows the inspection status overview from June 1, 2019 to June 26,2019 . The status is divided into three types: series common and normal. From the figure, we can see that the equipment clock in the series state of the power grid system is kept in a very small state, and the maximum value is $10 \%$ of 2019 / 6 / 1 . Although the proportion of common state equipment was the largest at the beginning, reaching about $65 \%$ at $2019 / 6 / 1$, it decreased to $35 \%$ at the later stage, accompanied by the increase of the proportion of normal equipment, from $25 \%$ at

\section{Alarm classification overview}

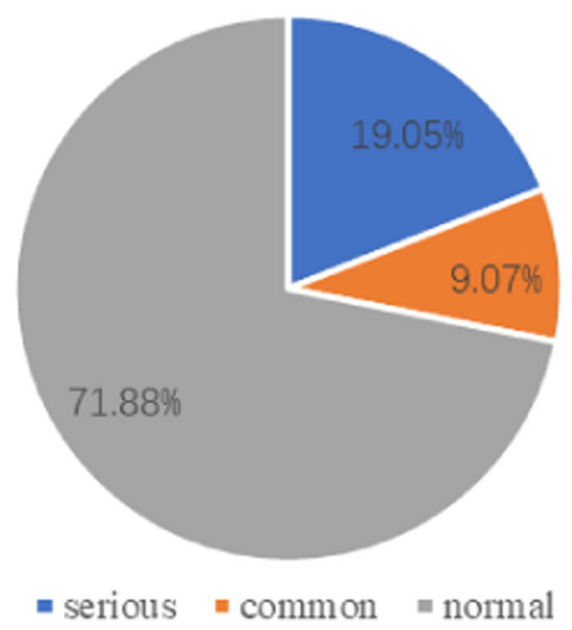

Fig. 8 Alarm classification overview the beginning to $55 \%$ later. From the time point of view, the number of series and common state equipment is gradually decreasing while the number of normal state equipment is gradually increasing, which shows that the number of unavailable equipment can be greatly reduced and the operation efficiency of the power grid system can be improved through the flexible automatic detection system of the power grid. At the same time, the number of equipment in series state is slowly reduced, which shows that the operation quality of power grid is slowly improving, and also shows the excellent performance of our power grid flexible automatic detection system. The historical alarms overview demonstrates the system's ability of recording the historical running status of the power grid in the form of a diagram, which accommodates the dispatchers with a visual review of the variation trend of the status of the power grid. Our method has a remarkable effect in dealing with the flexible automatic network detection. It can be seen from the experiment that this kind of network greatly reduces the running time of detection and avoids a lot of complicated process of manual inspection. The real and detailed use of data also reflects the high efficiency of this method in data analysis for security management of dispatching data network.

\section{Conclusion}

In this paper, the automatic power grid dispatching system was put forward to replace the currently adopted manual management of electric power system. The automatic power grid dispatching system proposed in this paper provides customers with security preservation, operation data acquisition, business data reporting, and supports in scheduling and decision-making. The experimental results show that the proposed system is effective and efficient in power grid dispatching. 


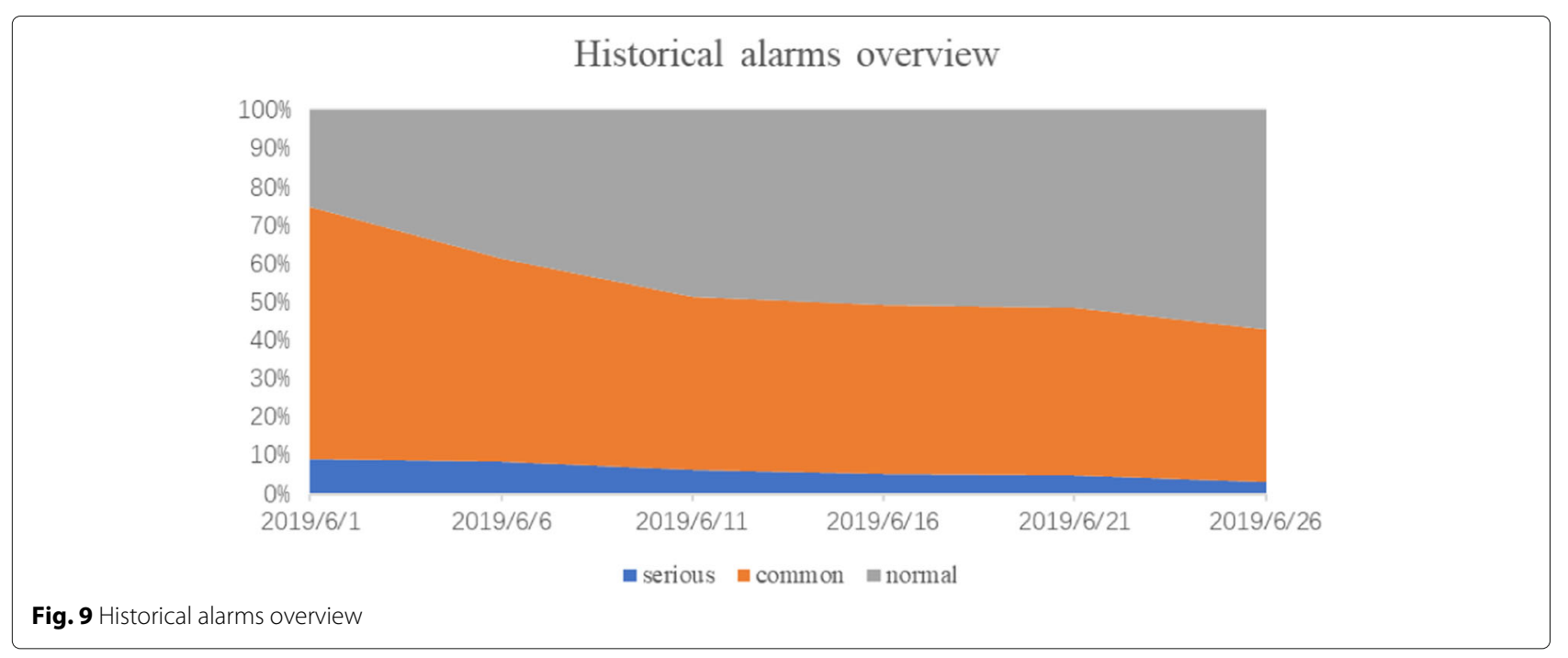

\section{Acknowledgements}

Not applicable.

\section{Authors' contributions}

Xianrui Yang, Yuming Liu, Jiehong Wang, Zhao Yao, Yanping Zhou and Shucun Fu conceived and designed the study. Xianrui Yang and Shucun Fu performed the simulations. Yuming Liu, Jiehong Wang, Zhao Yao and Yanping Zhou wrote the paper. All authors reviewed and edited the manuscript. All authors read and approved the final manuscript.

\section{Funding}

No Funding.

\section{Availability of data and materials}

The raw data required to reproduce these findings cannot be shared at this time as the data also forms part of an ongoing study.

\section{Competing interests}

The authors declare that they have no competing interests.

\section{Author details}

${ }^{1}$ Kunming Power Supply Bureau of Yunnan Power Grid Co., Ltd, 650041 Kunming, China. ${ }^{2}$ Nanjing University of Information Science and Technology, China No.219 Ningliu Road, 210044 Nanjing, China.

\section{Received: 20 January 2020 Accepted: 27 February 2020}

\section{Published online: 09 April 2020}

\section{References}

1. Poolla BK, Bolognani S, Dörfler F (2017) Optimal placement of virtual inertia in power grids. IEEE Trans Autom Control 62(12):6209-6220

2. Kanna S, Moniri A, Xia Y, Constantinides AG, Mandic DP (2019) A data analytics perspective of power grid analysis-part 2: Teaching old power systems new tricks [lecture notes]. IEEE Signal Proc Mag 36(3):110-117

3. Zhou J, Hu XS, Ma Y, Sun J, Wei T, Hu S (2019) Improving availability of multicore real-time systems suffering both permanent and transient faults. IEEE Trans Comput 68(12):1785-1801

4. Morello R, Mukhopadhyay SC, Liu Z, Slomovitz D, Samantaray SR (2017) Advances on sensing technologies for smart cities and power grids: A review. IEEE Sensors J 17(23):7596-7610

5. Xu X, Liu X, Xu Z, Dai F, Zhang X, Qi L (2019) Trust-oriented iot service placement for smart cities in edge computing. IEEE Internet Things J:1 https://doi.org/10.1109/jiot.2019.2959124

6. Chen Z, Wu J, Xia Y, Zhang X (2017) Robustness of interdependent power grids and communication networks: A complex network perspective. IEEE Trans Circ Syst II Express Briefs 65(1):115-119
7. Duan J, Shi D, Diao R, Li H, Wang Z, Zhang B, Bian D, Yi Z (2019) Deep-reinforcement-learning-based autonomous voltage control for power grid operations. IEEE Trans Power Syst

8. Zhang Y, Wang K, He Q, Chen F, Deng S, Zheng Z, Yang Y (2019) Covering-based web service quality prediction via neighborhood-aware matrix factorization. IEEE Trans Serv Comput

9. Wu R, Luo H, Qi R, Gao M, Liang C (2019) Study on the development direction of power grid dispatching technical support system, Vol. 260. https://doi.org/10.1051/matecconf/201926001004

10. Wang T, Ke H, Zheng X, Wang K, Sangaiah AK, Liu A (2019) Big data cleaning based on mobile edge computing in industrial sensor-cloud. IEEE Trans Ind Inform

11. Xu X, Fu S, Qi L, Zhang X, Liu Q, He Q, Li S (2018) An iot-oriented data placement method with privacy preservation in cloud environment. J Netw Comput Appl 124:148-157

12. Liu H, Kou H, Yan C, Qi L (2019) Link prediction in paper citation network to construct paper correlation graph. EURASIP J Wirel Commun Netw 2019(1):1-12

13. Xin Y, Shi J, Zhou J, Gao Z, Tao H, Shang X, Zhai M, Guo J, Yang S, Nan G, et al. (2015) Technology development trends of smart grid dispatching and control systems. Autom Electr Power Syst 39(1):2-8

14. Qiu W, Guo Z, Chen J, Zeng S, Sun Y (2019) Power system data management design. In: 2018 International Conference on Mathematics, Modeling, Simulation and Statistics Application (MMSSA 2018). Atlantis Press

15. Zhou G-P, Luo H-H, Ge W-C, Ma Y-L, Qiu S, Fu L-N (2019) Design and application of condition monitoring for power transmission and transformation equipment based on smart grid dispatching control system. J Eng 2019(16):2817-2821

16. Qi L, Chen Y, Yuan Y, Fu S, Zhang X, Xu X (2019) A qos-aware virtual machine scheduling method for energy conservation in cloud-based cyber-physical systems. World Wide Web. https://doi.org/10.1007/ s11280-019-00684-y

17. Zhang Y, Yin C, Wu Q, He Q, Zhu H, IEEE Trans Syst Man Cybern Syst (2019) Location-aware deep collaborative filtering for service recommendation:1-12. https://doi.org/10.1109/tsmc.2019.2931723

18. Zhang S, Choo K-KR, Liu Q, Wang G (2018) Enhancing privacy through uniform grid and caching in location-based services. Futur Gener Comput Syst 86:881-892

19. Chen J. (2019) Research on power system automation communication technology for smart grid. In: IOP Conference Series: Materials Science and Engineering. IOP Publishing Vol. 569. p 042025. https://doi.org/10. 1088/1757-899x/569/4/042025

20. Xu X, Liu X, Xu Z, Wang C, Wan S, Yang X (2019) Joint optimization of resource utilization and load balance with privacy preservation for edge services in $5 \mathrm{~g}$ networks. Mob Netw Appl:1-12 
21. Zhang S, Wang G, Bhuiyan MZA, Liu Q (2018) A dual privacy preserving scheme in continuous location-based services. IEEE Internet Things J 5(5):4191-4200

22. Gong W, Qi L, Xu Y (2018) Privacy-aware multidimensional mobile service quality prediction and recommendation in distributed fog environment. Wirel Commun Mob Comput 2018:1-8. https://doi.org/10.1155/2018/ 3075849

23. Zhang Y, Cui G, Deng S, Chen F, Wang Y, He Q (2018) Efficient query of quality correlation for service composition. IEEE Trans Serv Comput:1. https://doi.org/10.1109/tsc.2018.2830773

24. Xu X, Mo R, Dai F, Lin W, Wan S, Dou W (2019) Dynamic resource provisioning with fault tolerance for data-intensive meteorological workflows in cloud. IEEE Trans Ind Inform:1. https://doi.org/10.1109/tii. 2019.2959258

25. Xu X, Zhang X, Khan M, Dou W, Xue S, Yu S (2017) A balanced virtual machine scheduling method for energy-performance trade-offs in cyber-physical cloud systems. Futur Gener Comput Syst 105:789-799. https://doi.org/10.1016/j.future.2017.08.057

26. Tingting F, Junqi Z, Guangjin P, Wenrui X (2019) Research on discharging price of electric vehicle participating in power grid dispatching considering reverse cost. IOP Conf Ser Earth Environ Sci 267:042054. https://doi.org/10.1088/1755-1315/267/4/042054

27. Xu X, He C, Xu Z, Qi L, Wan S, Bhuiyan MZA (2019) Joint optimization of offloading utility and privacy for edge computing enabled iot. IEEE Internet of Things J. https://doi.org/10.1109/jiot.2019.2944007

28. Okami S, Kohtake N (2017) Modeling and analysis of health-information system of systems for managing transitional complexity using engineering systems multiple-domain matrix. In: 2017 Annual IEEE International Systems Conference (SysCon). pp 1-8. https://doi.org/10. 1109/SYSCON.2017.7934805

29. Qin X, Ying W (2015) Design of explosive production information and managing system based on internet of things. In: 2015 International Conference on Control, Automation and Robotics. pp 173-176. https:// doi.org/10.1109/ICCAR.2015.7166025

30. Levendovszky T, Dubey A, Otte WR, Balasubramanian D, Coglio A, Nyako S, Emfinger W, Kumar P, Gokhale A, Karsai G (2014) Distributed real-time managed systems: A model-driven distributed secure information architecture platform for managed embedded systems. IEEE Softw 31(2):62-69. https://doi.org/10.1109/MS.2013.143

31. Dmitriev SA, Kokorin EL, Volobuev AV, Korelina AA (2017) The object-oriented model of the electrical equipment failures risks assessment. In: 2017 IEEE 58th International Scientific Conference on Power and Electrical Engineering of Riga Technical University (RTUCON). pp 1-5. https://doi.org/10.1109/RTUCON.2017.8124790

32. Hill Cl, Areerak K, Yang T, Bozhko S, Odavic M (2015) Automated stability assessment of more electric aircraft electrical power systems. In: 2015 International Conference on Electrical Systems for Aircraft, Railway, Ship Propulsion and Road Vehicles (ESARS). pp 1-6. https://doi.org/10.1109/ ESARS.2015.7101426

33. Kryukov OV, Blagodarov DA, Dulnev NN, Safonov YM, Fedortsov NN, Kostin AA (2018) Intelligent control of electric machine drive systems. In: 2018 X International Conference on Electrical Power Drive Systems (ICEPDS). pp 1-4. https://doi.org/10.1109/ICEPDS.2018.8571670

34. Zhou J, Sun J, Zhou X, Wei T, Chen M, Hu S, Hu XS (2018) Resource management for improving soft-error and lifetime reliability of real-time mpsocs. IEEE Trans Comput Aided Des Integr Circ Syst 38(12):2215-2228

35. Zhou J, Sun J, Cong P, Liu Z, Zhou X, Wei T, Hu S (2019) Security-critical energy-aware task scheduling for heterogeneous real-time mpsocs in iot. IEEE Trans Serv Comput

36. Zhang S., Li X., Tan Z., Peng T., Wang G. (2019) A caching and spatial k-anonymity driven privacy enhancement scheme in continuous location-based services. Futur Gener Comput Syst 94:40-50

37. Qi L, Dou W, Zhang X, Yu S (2017) Amplified locality-sensitive hashing for privacy-preserving distributed service recommendation. In: Security, Privacy, and Anonymity in Computation, Communication, and Storage. Springer. pp 280-297. https://doi.org/10.1007/978-3-319-72389-1_23

38. Qi L, Zhang X, Li S, Wan S, Wen Y, Gong W (2020) Spatial-temporal data-driven service recommendation with privacy-preservation. Inf Sci 515:91-102

39. Cao H, Xu X, Liu Q, Xue Y, Qi L (2019) Uncertainty-aware resource provisioning for workflow scheduling in edge computing environment.
In: 2019 18th IEEE International Conference On Trust, Security And Privacy In Computing And Communications/13th IEEE International Conference On Big Data Science And Engineering (TrustCom/BigDataSE). IEEE. pp 734-739. https://doi.org/10.1109/trustcom/bigdatase.2019.00105

40. Xu X, Cao H, Geng Q, Liu X, Dai F, Wang C (2020) Dynamic resource provisioning for workflow scheduling under uncertainty in edge computing environment. Concurr Comput Pract Experience. https://doi. org/10.1002/cpe.5674

41. Xu X, Zhang X, Gao H, Xue Y, Qi L, Dou W (2019) Become: Blockchainenabled computation offloading for iot in mobile edge computing. IEEE Trans Ind Inform:1-1. https://doi.org/10.1109/tii.2019.2936869

\section{Publisher's Note}

Springer Nature remains neutral with regard to jurisdictional claims in published maps and institutional affiliations.

\section{Submit your manuscript to a SpringerOpen ${ }^{\circ}$ journal and benefit from:}

- Convenient online submission

- Rigorous peer review

- Open access: articles freely available online

- High visibility within the field

- Retaining the copyright to your article

Submit your next manuscript at $>$ springeropen.com 